\title{
Orbital ordering and one-dimensional magnetic correlation in vanadium spinel oxides $A \mathrm{~V}_{2} \mathrm{O}_{4}(A=\mathrm{Zn}, \mathrm{Mg}$, or $\mathrm{Cd})$
}

\author{
Yukitoshi Motome a,1,* Hirokazu Tsunetsugu b,1 \\ ${ }^{a}$ RIKEN (The Institute of Physical and Chemical Research), Saitama 351-0198, Japan \\ ${ }^{\mathrm{b}}$ Yukawa Institute for Theoretical Physics, Kyoto University, Kyoto 606-8502, Japan
}

\begin{abstract}
We present our theoretical results on the mechanism of two transitions in vanadium spinel oxides $A \mathrm{~V}_{2} \mathrm{O}_{4}(A=\mathrm{Zn}$, $\mathrm{Mg}$, or $\mathrm{Cd}$ ) in which magnetic $\mathrm{V}$ cations constitute a geometrically-frustrated pyrochlore structure. We have derived an effective spin-orbital-lattice coupled model in the strong correlation limit of the multiorbital Hubbard model, and applied Monte Carlo simulation to the model. The results reveal that the higher-temperature transition is a layered antiferro-type orbital ordering accompanied by tetragonal Jahn-Teller distortion, and the lower-temperature transition is an antiferromagnetic spin ordering. The orbital order lifts the magnetic frustration partially, and induces spatial anisotropy in magnetic exchange interactions. In the intermediate phase, the system can be considered to consist of weakly-coupled antiferromagnetic chains lying in the perpendicular planes to the tetragonal distortion.
\end{abstract}

Key words:

vanadium spinel oxides, geometrical frustration, orbital ordering, antiferromagnetic ordering, Monte Carlo simulation

Vanadium spinel oxides $A \mathrm{~V}_{2} \mathrm{O}_{4}$ with nonmagnetic cations $A=\mathrm{Zn}, \mathrm{Mg}$, or $\mathrm{Cd}$ are one of the most typical geometrically-frustrated systems; the magnetic $\mathrm{V}$ cations constitute the pyrochlore lattice, which is a three-dimensional network of corner-sharing tetrahedra. The geometrical frustration strongly suppresses development of magnetic correlations, and the antiferromagnetic $(\mathrm{AF})$ ordering sets in at a much lower temperature $\sim 40 \mathrm{~K}$ than the Curie-Weiss temperature $\sim 1000 \mathrm{~K}[1]$. Besides the AF transition, at a slightly higher temperature $\sim 50 \mathrm{~K}$, the system shows another phase transition with the structural change from high-

\footnotetext{
* Corresponding Author: RIKEN, 2-1 Hirosawa, Wako, Saitama 351-0198, Japan. Phone: +81-48-467-1379, Fax: +8148-467-1339, Email: motome@riken.jp

1 This work is supported by a Grant-in-Aid and NAREGI from the Ministry of Education, Science, Sports, and Culture. A part of the work was accomplished during Y. M. was staying at the Yukawa Institute of Theoretical Physics, with the support from The 21st Century for Center of Excellence program, 'Center for Diversity and Universality in Physics'.
}

temperature cubic to low-temperature tetragonal symmetry [1]. The origin of the two successive transitions is under debate. In particular, theories based on spin degree of freedom only or the spin-lattice coupling appear to be insufficient to describe the two transitions in a comprehensive manner [2].

In the present study, we focus on the $t_{2 g}$ orbital degree of freedom in these vanadium spinels to understand the microscopic mechanism of the two successive transitions. Each $\mathrm{V}^{3+}$ cation has two $3 d$ electrons in threefold $t_{2 g}$ orbitals, and therefore the orbital degree of freedom is active. Starting from the standard multiorbital Hubbard model, we consider the perturbation in the strong correlation limit to describe the low energy physics of these insulating materials, and derive the effective spin-orbital-lattice coupled model in the form [2]

$$
\begin{aligned}
& H=H_{\mathrm{SO}}+H_{\mathrm{JT}}, \\
& H_{\mathrm{SO}}=-J \sum_{\langle i, j\rangle} h_{i j}-J_{3} \sum_{\langle i, j\rangle\rangle} h_{i j},
\end{aligned}
$$




$$
\begin{aligned}
h_{i j}= & \left(A+B \boldsymbol{S}_{i} \cdot \boldsymbol{S}_{j}\right)\left[n_{i \alpha(i j)} \bar{n}_{j \alpha(i j)}+\bar{n}_{i \alpha(i j)} n_{j \alpha(i j)}\right] \\
& +C\left(1-\boldsymbol{S}_{i} \cdot \boldsymbol{S}_{j}\right) n_{i \alpha(i j)} n_{j \alpha(i j)} \\
H_{\mathrm{JT}} & =\gamma \sum_{i} Q_{i} \epsilon_{i}+\sum_{i} Q_{i}^{2} / 2-\lambda \sum_{\langle i, j\rangle} Q_{i} Q_{j}
\end{aligned}
$$

where $\boldsymbol{S}_{i}$ is the $S=1$ spin operator and $n_{i \alpha}$ is the density operator for site $i$ and orbital $\alpha=1\left(d_{y z}\right), 2\left(d_{z x}\right)$, $3\left(d_{x y}\right)$. Here, $\bar{n}_{i \alpha}=1-n_{i \alpha}$ and $\epsilon_{i}=n_{i 1}+n_{i 2}-2 n_{i 3}$; and we impose a local constraint $\sum_{\alpha=1}^{3} n_{i \alpha}=2$ at each site. The summations with $\langle i, j\rangle$ and $\langle\langle i, j\rangle\rangle$ are taken over the nearest-neighbor (NN) sites and thirdneighbor sites, respectively. Here, we take into account only the dominant $\sigma$-bond hopping integrals in the original multiorbital Hubbard model, which results in the orbital diagonal interaction in $H_{\mathrm{SO}} ; \alpha(i j)$ is the orbital which gives rise to the $\sigma$ bond between sites $i$ and $j . H_{\mathrm{JT}}$ describes the orbital-lattice coupling part, where $\gamma$ is the electron-phonon coupling constant of the tetragonal Jahn-Teller (JT) mode, $Q_{i}$ denotes the amplitude of local lattice distortion at site $i$, and $\lambda$ describes the interaction between NN JT distortions, which mimics the cooperative aspect of the JT distortion. The parameters in $H_{\mathrm{SO}}$ are given by the coupling constants in the starting multiorbital Hubbard Hamiltonian, and we use the reasonable estimates as $J_{3} / J=$ 0.02 with $J \simeq 200 \mathrm{~K}, A=1.21, B=0.105$, and $C=$ 0.931 . For the JT parameters, we take $\gamma^{2} / J=0.04$ and $\lambda / J=0.15$, which are typical values to reproduce the tetragonal distortion observed in experiments.

We have performed Monte Carlo (MC) simulation to investigate thermodynamic properties of the model (1), which is a classical simulation to avoid the negative sign problem due to the geometrical frustration. Since quantum nature exists only in the spin operators in $H_{\mathrm{SO}}$, we approximate them by classical vectors, and apply a standard metropolis MC algorithm. Details of MC calculations will be reported elsewhere [3].

MC results show that the model (1) exhibits two transitions: One is first order transition at $T_{\mathrm{O}} \simeq 0.19 \mathrm{~J}$ corresponding to the orbital ordering with the tetragonal JT distortion, and the other is at $T_{\mathrm{N}} \simeq 0.115 \mathrm{~J}$ with continuous growth of the AF spin ordering. The orbital order below $T_{\mathrm{O}}$ is a layered antiferro type; $\left(d_{x y}\right.$, $\left.d_{y z}\right)$ and $\left(d_{x y}, d_{z x}\right)$-occupied planes stack alternatively in the $c$ direction ( $c$ is the axis of the tetragonal distortion). This orbital ordering plays a crucial role to reduce the magnetic frustration in the following way: Let us consider an effective spin Hamiltonian by replacing the orbital part in $H_{\mathrm{SO}}$ by its mean-field. The model has highly anisotropic spin exchanges; for instance, between $\mathrm{NN}$ sites, the strong $\mathrm{AF}$ interaction $J_{\mathrm{AF}} \simeq$ $0.931 J$ in the $x y$ direction whereas the weak ferromagnetic interaction $J_{\mathrm{F}} \simeq-0.105 J$ in the $y z$ and $z x$ directions. Moreover, the weak ferromagnetic interactions $J_{\mathrm{F}}$ are completely frustrated between the AF $x y$ chains

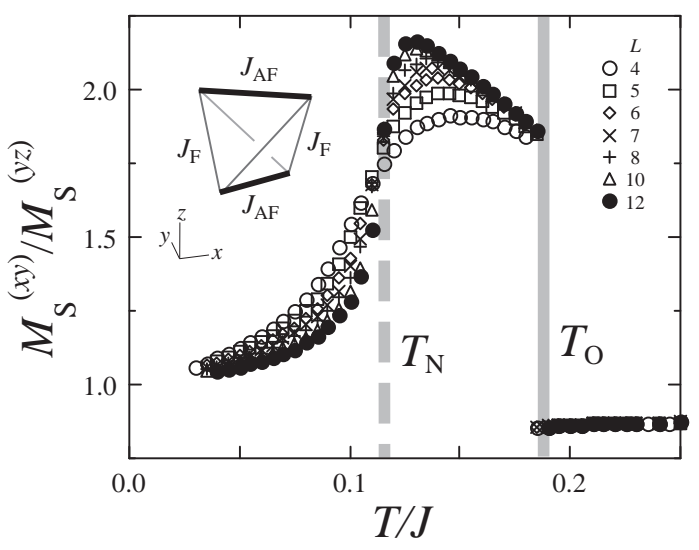

Fig. 1. Monte Carlo results of the anisotropy between the staggered moments in the $x y$ chains and in the $y z$ chains. The orbital ordering temperature $T_{\mathrm{O}}$ and the antiferromagnetic spin ordering temperature $T_{\mathrm{N}}$ are shown. The system size is $L^{2} \times 16$ sites. Inset: Anisotropic spin exchange interactions under the orbital ordering.

due to the geometry of the pyrochlore structure. These anisotropic exchange interactions are shown in the inset of Fig. 1 in a tetrahedron unit. As a consequence, the magnetic correlation becomes highly anisotropic, and the system looks like a weakly-coupled AF chains. Figure 1 shows the ratio between the staggered moments along the $x y$ chains and along the $y z(z x)$ chains. Note that the latter moment corresponds to the ordering structure with periodicity four along the $y z(z x)$ chains. Above $T_{\mathrm{N}}$, both moments are zero in the limit of $L \rightarrow \infty$, and therefore the ratio gives information on the anisotropy of the magnetic correlation length. The result shows that the anisotropy is suddenly enhanced below $T_{\mathrm{O}}$ and takes a large value for $T_{\mathrm{N}}<T<T_{\mathrm{O}}$.

Below $T_{\mathrm{N}}$, the AF $x y$ chains are aligned by the thirdneighbor interaction $J_{3}$ to form the three-dimensional AF order, whose pattern is consistent with the neutron scattering results [4]. There, the ratio in Fig. 1 approaches 1 in the limit of $L \rightarrow \infty$ since both moments are finite and the same.

The one-dimensional anisotropy in the intermediate phase $T_{\mathrm{N}}<T<T_{\mathrm{O}}$ can be observed, for example, by neutron scattering measurement. Such experiment is desired to confirm our scenario.

\section{References}

[1] Y. Ueda, N. Fujiwara, and H. Yasuoka, J. Phys. Soc. Jpn. 66, 778 (1997), and references therein.

[2] H. Tsunetsugu and Y. Motome, Phys. Rev. B 68, 060405(R) (2003), and references therein.

[3] Y. Motome and H. Tsunetsugu, preprint (cond-mat/0406039).

[4] S. Niziol, Phys. Status Solidi A 18, K11 (1973). 